Estudios sobre el Mensaje Periodístico ISSN-e: 1988-2696

http://dx.doi.org/10.5209/ESMP.55598

\title{
Siguiendo al flautista. Framing del independentismo catalán en las votaciones de 2014 y 2015
}

\author{
Carlos A. Ballesteros Herencia ${ }^{1}$
}

Recibido: 19 de marzo de 2016 / Aceptado: 16 de septiembre de 2016

Resumen. El debate sobre la independencia de Cataluña se convirtió en el tema principal de las elecciones autonómicas catalanas del 27 de septiembre de 2015. Un año antes, el 9 de noviembre de 2014, se había celebrado una polémica consulta sobre idéntico asunto. En esta investigación se contrastaron las representaciones informativas de ambas convocatorias electorales realizadas por los principales periódicos de Madrid y Cataluña. Se efectuó un análisis de contenido de carácter cuantitativo para calcular estadísticos descriptivos e inferenciales, que mostraron la relevancia máxima concedida a este tema durante el tiempo que duró la campaña electoral. El debate sobre la independencia supuso más del $80 \%$ de las informaciones sobre la campaña electoral. Además se detectó la novedosa aparición de fuentes y protagonistas económicos e internacionales respecto a la consulta del año anterior. En cambio se mantuvo el enmarcado informativo antagónico entre los medios catalanes y madrileños.

Palabras clave: Información política; marcos informativos; referéndum; Cataluña; 9-N.

\section{[en] Following the Pied Piper. Framing of the Catalan independence movement in 2014 and 2015 votings}

\begin{abstract}
The debate about the independence of Catalonia became the main theme of the Catalonian regional elections of 27 September 2015. A year earlier, on November 9, 2014, there had been a controversial referendum on the same subject. In this research the informative representations of both electoral calls made by major newspapers of Madrid and Catalonia were contrasted. A quantitative content analysis was conducted to calculate descriptive and inferential statistics, which showed the utmost importance given to this issue during the period of the election campaign. Besides the emergence of new sources, such as economic and international actors, were detected. The antagonistic news framing among the Catalonian and Madrid newspapers remained.
\end{abstract}

Keywords: Political news; news frames; framing; Cataluña.

Sumario. 1. Introducción; 1.1. Aplicación de la teoría del framing a la investigación de la información política; 1.2. El carácter plebiscitario de las elecciones autonómicas del 27 de septiembre de 2015; 1.3. Objetivos; 1.4. Hipótesis; 1.5. Preguntas de investigación. 2. Metodología; 2.1. Diseño y muestra; 2.2. Codificación de la muestra; 2.3. Procedimiento y fiabilidad. 3. Resultados. 4. Conclusiones. 5. Referencias bibliográficas.

Cómo citar: Ballesteros Herencia, Carlos A. (2017): "Siguiendo al flautista. Framing del independentismo catalán en las votaciones de 2014 y 2015”, en Estudios sobre el Mensaje Periodístico 23 (1), 307-323.

Universidad de Valladolid

E-mail: cballesteros@hmca.uva.es 


\section{Introducción}

\subsection{Aplicación de la teoría del framing a la investigación de la información política}

El estudio de los marcos se ha expandido rápidamente en las últimas décadas (Matthes y Kohring, 2008; Weaver, 2007) hasta convertirse en una de las más populares áreas de investigación para los investigadores de la comunicación (Cacciatore, Scheufele \& Iyengar, 2015; Riffe, 2004; Bryant y Miron, 2004; Sádaba et al., 2012). Dentro de estos estudios, Muñiz (2015) señala la muy destacada presencia de los trabajos centrados en la información sobre política, en especial en las campañas electorales. Así, Miller y Riechert (2001) entienden la campaña como una batalla de enmarcado entre partidos que procuran imponer su propio diagnóstico de la situación.

La definición fundacional de este concepto, dentro del campo de la sociología, suele remitirse a Goffman (1974: 10), cuando señala que "la definición de una situación [...] se construye de acuerdo con los principios de organización que gobiernan los hechos - al menos los sociales-y nuestra implicación subjetiva en ellos". Entman (1993: 52) ofrece la definición más extendida de marco: "Enmarcar (to frame) es seleccionar algunos aspectos de una realidad percibida y hacerlos más destacados en un texto comunicativo, de tal forma que promueva una definición particular del problema, una interpretación de las causas, una evaluación moral, y/o una recomendación de tratamiento para el asunto descrito".

Por su parte, Gitlin (1980: 6) define marcos como "los principios de selección, enfásis y presentación compuestos de pequeñas teorías tácitas sobre lo que existe, lo que ocurre y lo que pasa". Mientras, Gamson y Modigliani (1987: 143) consideran a los marcos una idea organizadora central o una trama que provee de significado a una serie de hechos, generando una conexión entre ellos".

Precisamente Matthes (2009) enumera entre las definiciones más citadas de marco mediático, las referidas de Entman (1993), Gamson \& Modigliani (1987) y Gitlin (1980), seguidas por las de Iyengar (1991), Semetko y Valkenburg (1999) y Goffman (1977), pero advirtiendo de que citar una definición "no significa necesariamente que sea usada como una guía directa para la operacionalización" (p. 354). De este modo, la translación de una definición de framing a pasos concretos y operativos no es transparente en una enorme parte de la literatura (p.359).

Además Scheufele \& Iyengar (2014: 10) precisan que si bien el trabajo de Entman $(1991,1993)$ tuvo "un poderoso efecto catalizador preparando el escenario para numerosos estudios sobre framing en los siguientes treinta años", al mismo tiempo esta omnicomprensiva definición del framing "también ayudó a establecer el escenario para la vaguedad conceptual que rodea el concepto de framing".

La investigación de los marcos mediáticos (news frames) requiere por tanto de una metodología acorde a la línea teórica en que se sustenta, de tal modo que una operativa transparente opere como motor de unos resultados derivados de un proceso coherente y fundamentado.

La presente investigación expone cada paso metodológico como traducción directa de una concreta perspectiva teórica. Partiendo de la definición del término anglosajón frame como "la estructura en la que algo está construido" (Longman, 1993: 206), el estudio de la sintaxis, en tanto "parte del lenguaje que estudia la estructura de la oración" (Larousse, 2007: 930) se revela esencial a la hora de estudiar los 
mensajes inmersos en los textos periodísticos. En esta línea, Lind y Salo (2002: 215) estiman que los marcos deben ser identificados mediante el estudio del lenguaje empleado por los medios. Mientras que D'Angelo (2002: 881) incluye a las estructuras sintácticas entre los dispositivos de enmarcado ("framing devices").

Se parte de la estructura sintáctica más elemental de los textos periodísticos, al identificar el sujeto (actor protagonista de la acción) y el predicado (acción descrita) de cada noticia. Además, se utilizan estos componentes sintácticos básicos para definir la variable "hecho noticioso" en función del actor y la acción de cada información, siguiendo una línea metodológica de investigación ya aplicada a diversas cuestiones informativas (Ballesteros, 2014, 2015a, 2015b y 2015c).

Así, se toma como eje la definición de frame que proponen Igartua, Muñiz \& Cheng (2005: 158) en tanto elección, énfasis o importancia atribuida a diferentes elementos (entre otros, los actores protagonistas y acciones realizadas por / sobre ellos, y los atributos asociados al objeto de estudio) y la forma cómo covarían dichos elementos más o menos enfatizados en un texto.

También Matthes y Kohring (2008: 264) desarrollan esta línea teórica y metodológica al afirmar que un marco consiste en varios elementos de enmarcado, y cada elemento de enmarcado consiste en varias variables analíticas de contenido. "Algunas de estas variables se agrupan sistemáticamente de un modo específico, formando de este modo un patrón determinado. En otras palabras, cada marco está caracterizado por un patrón específico de variables".

Una importante función de los marcos es la de evaluar la información que transportan (Entman, 1993; Matthes y Kohring, 2008; Neuman et al., 1992; Tábara y Costejá, 2001), por lo que en este trabajo se ha estudiado qué evaluación del acontecimiento principal o "hecho noticioso" se realiza en cada noticia.

Finalmente, una variable esencial en el estudio de los mensajes comunicativos ha sido la fuente de información ya desde Laswell (1948) en su conocida frase "Quién dice qué, por qué canal, a quién y con qué efectos “. Desde una óptica más cercana al concepto de news frames, Luhman (2000: 154) describe los esquemas o scripts como estructuras que responden a las estimulaciones provenientes de determinadas fuentes, mientras que Porto (2002: 2) se interesa por el promotor o "esponsor" de los "marcos interpretativos" de las cuestiones políticas. También Entman (1993: 52) cita la presencia o ausencia de las fuentes de información como indicador de los marcos presentes en el texto.

\subsection{El carácter plebiscitario de las elecciones autonómicas del 27 de septiembre de 2015}

Las elecciones autonómicas del 27 de septiembre de 2015 estuvieron marcadas desde el mismo momento de su convocatoria, por su pretendido carácter plebiscitario, esto es, su conversión en una consulta popular sobre la independencia de Cataluña. El presidente de la Generalitat de Cataluña, Artur Mas, firmó el 3 de agosto de 2015 el decreto de convocatoria, afirmando que se trataba de un «último recurso» ante la imposibilidad de celebrar "un referéndum al uso" (Sastre, 2015). Según el presidente catalán, estas elecciones autonómicas debían "servir para responder a la pregunta de si los catalanes quieren un Estado independiente" (Noguer, 2015).

Utilizando la expresión de El Confidencial (2015) esta convocatoria dividió de inmediato a los partidos políticos catalanes "en dos frentes: el de los que creen que 
las elecciones del próximo 27 de septiembre se estará preguntando a los catalanes por la independencia y el de los que creen que no". De un lado, tanto la coalición "Catalunya sí que es pot" —-formada por Iniciativa per Catalunya Verds, Esquerra Unida i Alternativa, Podemos y Equo-- como el Partit dels Socialistes de Catalunya (PSC), Partido Popular y Unió Democrática de Cataluña (UDC) coincidieron en rechazar el carácter plebiscitario de las elecciones del 27-S (García, 2015); del otro, la plataforma "Junts pel sí” que aunaba a Convergencia Democrática de Cataluña y Esquerra Republicana, y el partido asambleario Candidatura de Unidad Popular (CUP).

Sin embargo, entre los propios partidos que negaban el carácter "plebiscitario" del 27-S, algunos reconocían lo extraordinario de la cita electoral, como el líder del Partido Popular de Cataluña, Xavier García Albiol, al asegurar que serían "las autonómicas más importantes de la historia", porque no estaba "en juego un eje izquierda-derecha, sino que en el Parlament haya o no una mayoría independentista” (EFE, 2015). Otros, como el candidato de Cataluña sí que es pot, Lluís Rabell, afirmaban que serían "unas elecciones normales, unas autonómicas más" (El Confidencial, 2015).

Según el catedrático de Derecho Constitucional de la Universidad del País Vasco (UPV-EHU) Eduardo Vírgala, no se trataría de "unas elecciones normales en las que se lleva un programa con 200 puntos, sino que se llevaría un programa en el que solo hay un punto o todo lo demás sería completamente accesorio". De este modo, se pretendería transmitir a la opinión pública "que no está eligiendo un Parlamento, sino decidiendo que ese Parlamento inmediatamente va a tomar una decisión trascendental, como podría ser una declaración de independencia" (Pérez, 2014).

Los programas electorales de los partidos que concurrieron a las elecciones también recogieron este debate de distinto modo. Así el programa de Junts pel sí estaba confeccionado desde el principio hasta el final partiendo de esta idea: su preámbulo titulado "El País que queremos" daba paso un primer bloque "La hoja de ruta hacia la independencia", un segundo bloque donde se describían "Las estructuras de Estado", y un tercer y último "Cataluña, nuevo Estado de Europa". Junt pel sí presentaba las elecciones autonómicas como la "única opción para garantizar el derecho a decidir de los catalanes [...] a la que las formaciones políticas han de dar un carácter plebiscitario y que deben convertirse en la consulta que el Estado español ha impedido que se pudiera hacer" (Junt pel sí, 2015: 29). A continuación detallaba que "si la ciudadanía de Cataluña escoge, a través de las elecciones del 27-S, una mayoría de diputados a favor de la independencia, se iniciará un proceso hacia la creación de un Estado independiente" (2015: 30).

Por su parte, la CUP afirmaba en el primer párrafo de su programa electoral que “estas elecciones no serán unas elecciones cualquiera. El próximo 27-S no se escogerá un gobierno autonómico, sino que se decidirá si el pueblo de Cataluña quiere la independencia $y$, en consecuencia, iniciar un proceso constituyente para decidir cómo debe ser El País que debemos construir entre todos y todas. Por ello, la CUP — Crida Constituent ha elaborado un programa plebiscitario" (CUP, 2015: 3).

Del resto de partidos, sólo Catalunya sí que es pot se refería en su programa electoral al término "plebiscito", al manifestar que "a diferencia del concepto plebiscitario, defendemos que estas elecciones no tratan sólo de un tema, sino que queremos hablar de modelo social, económico, ecológico, y político; de mayorías de gobierno para hacer unas políticas socialmente justas, contrarias a los recortes de derechos y en las privatizaciones, e implacables en la lucha contra la corrupción y las malas praxis en las instituciones" (Catalunya sí que es pot, 2015: 171). Partido Popular, 
Partido Socialista, Ciudadanos y Unió no recogieron en su programa el término plebiscito.

Apenas un año antes, el 9 de noviembre de 2014 se había celebrado en Cataluña una consulta popular sobre la independencia de esta Comunidad Autónoma, denominada "proceso de participación ciudadana" tras la anulación por parte del Tribunal Constitucional (TC, 2014), de la convocatoria oficial de consulta (Decreto 129/2014, de 27 de septiembre, de la Generalitat de Catalunya). Esta convocatoria tuvo un importante reflejo en los medios de comunicación, estudiado en investigaciones anteriores (Ballesteros, 2015a y 2015b), en las que se describe una muy intensa cobertura mediática, así como diferentes modos de evaluar las acciones realizadas por cada sujeto en función de la procedencia geográfica de la prensa. "Mientras que los diarios de Madrid presentaron un proceso cargado de tintes negativos que proponía vulnerar la ley, los diarios de Barcelona relataron el normal funcionamiento político de las instituciones dentro de un Estado de Derecho" (Ballesteros, 2015a: 180).

Finalmente resulta interesante citar a Valera y López (2014: 44) cuando describen cómo en ocasiones la agenda de una campaña electoral puede centrarse sobre un único asunto como consecuencia de que "la realidad impone algunas restricciones de primer orden a la construcción de la factualidad política", para referirse a "prioridades empíricamente insoslayables", como guerras, atentados, desastres naturales o crisis económicas, que dejarían escasa capacidad a los partidos políticos para confeccionar libremente la agenda pública.

En cambio, en nuestro caso, la reducción del debate público y electoral a un punto único, la soberanía de Cataluña, no es consecuencia de factores ineludibles, sino de la elección estratégica de una parte del espectro político catalán, que de este modo trata de imponer el campo de juego más favorable a sus intereses.

\subsection{Objetivos}

1. Describir el contenido de los marcos informativos (news frames) de las elecciones autonómicas catalanas del 27 de septiembre de 2015.

2. Comparar el enmarcado informativo (news framing) de dos convocatorias electorales no solo consecutivas y cercanas temporalmente, sino temáticamente afines, y pretendidamente equivalentes pese a su muy diferente naturaleza legal.

\subsection{Hipótesis}

La representación informativa de la consulta sobre la independencia de Cataluña de 9 de noviembre de 2014 y de las elecciones autonómicas catalanas de 27 de septiembre de 2015 contiene rasgos tanto similares como diferenciadores, dada por un lado la afinidad argumental del debate político y, por otro, la diferente naturaleza legal de cada convocatoria.

\subsection{Preguntas de investigación}

Aplicando la línea teórica descrita tratamos de contestar a las siguientes preguntas de investigación en relación a nuestro objeto de estudio mediante una operativa metodológica acorde: 
1. ¿Cuáles son las fuentes de información más utilizadas durante la campaña electoral de las elecciones autonómicas catalanas del 27 de septiembre de 2015? ¿Son similares o diferentes a las empleadas en la consulta soberanista del 9 de noviembre de 2014 ?

2. ¿Cuáles son los principales actores y las principales acciones en el 27-S? ¿Son similares o diferentes a las empleadas el 9-N?

3. ¿Existen diferencias significativas entre el modo de evaluar las acciones realizadas por cada sujeto durante la campaña de las elecciones autonómicas en función de la procedencia geográfica de los medios analizados?

4. ¿Cómo se relacionan estos componentes en la composición del texto publicado?

5. ¿Utilizaron diferentes o similares marcos informativos los medios madrileños y catalanes sobre la consulta del 27-S?

\section{Metodología}

\subsection{Diseño y muestra}

Se llevó a cabo un análisis de contenido de todas las noticias publicadas durante la campaña electoral del 27 de septiembre de 2015 en la portada de los periódicos de información general de mayor audiencia de Madrid (El País, El Mundo, ABC) y de Cataluña (La Vanguardia, El Periódico, El Punt Avui), según el Estudio General de Medios más reciente (AIMC, 2015).

Se seleccionaron las informaciones aparecidas en las portadas de los periódicos citados, publicadas entre el 11 y el 25 de septiembre, periodo correspondiente con la campaña electoral de 2015 (Decreto 174/2015), referidas temáticamente a las elecciones autonómicas catalanas. Se analizó toda la población de estudio, que resultó en un total de 269 informaciones publicadas.

La unidad de análisis fue el titular principal de la información, y tan sólo cuando éste resultó difícil de codificar objetivamente por tratarse de una metáfora, un juego de palabras o una interpretación, se acudió a uno o varios subtítulos para interpretarlo. Esta elección tuvo como objeto centrar la investigación en el marco principal o dominante que cada periódico destacó cada día. Los titulares son considerados por Pan y Kosicki (1993: 59) como la señal más destacada "para activar determinados conceptos semánticamente relacionados en las mentes de los lectores”.

\subsection{Codificación de la muestra}

Para codificar cada una de las unidades de análisis, dado nuestro objetivo de comparar las citas electorales del 9 de noviembre de 2014 y del 27 de septiembre de 2015, se utilizó el mismo libro de códigos utilizado para investigar la consulta soberanista de 2014 (Ballesteros, 2015a y 2015b). De este modo las variables analizadas fueron:

a. Datos de identificación (número de unidad de análisis, fecha de publicación, texto del titular y texto del subtítulo).

b. Aspectos formales (orden de prioridad del titular, acompañamiento gráfico, aparición en portada, publicación en página impar). Se elaboró un índice de importancia propio, tomando como referentes los utilizados por Igartúa (2006) y Budd (1964 citado en Gutiérrez, 2001). 
c. Identificación de marcos informativos (fuente principal de la información, sujeto de la acción, campo semántico de la acción principal, hecho noticioso). Con esta última variable ("Hecho noticioso") se describió el acontecimiento descrito mediante la combinación de actor protagonista (sujeto) y acontecimiento descrito (predicado) de cada unidad de análisis, continuando la línea de investigaciones anteriores (Ballesteros, Dader y Muñiz, 2015; Ballesteros, 2014).

d. La noticia se refiere a la independencia de Cataluña.

e. Carácter evaluativo del acontecimiento principal. Se codificó como "carácter negativo", "carácter neutro o ambiguo" o "carácter positivo".

Todos estos atributos de la noticia fueron tratados como variables en un análisis estadístico con el que se trató de analizar la correlación existente entre ellos en términos estadísticamente significativos, siguiendo la línea de investigación descrita por Igartua, Muñiz y Cheng (2005).

\subsection{Procedimiento y fiabilidad}

Las unidades de análisis seleccionadas según los criterios descritos fueron codificadas mediante el programa SPSS v.20.0, que permitió calcular una serie de estadísticos descriptivos (p.e. medias, medianas, modas y desviaciones típicas) e inferenciales para la determinación de la asociación estadística y su significación entre variables (Tablas de Contingencia, chi cuadrado, prueba exacta, $V$ de Cramer).

La codificación de los datos fue realizada por un único juez, y con posterioridad un segundo juez realizó un nuevo análisis sobre una muestra del $20 \%$ de las unidades elegidas al azar (54 noticias), a fin de estimar la fiabilidad del proceso de codificación (intercoder reliability). Para dicha revisión se contó con la ayuda de la doctora ingeniera industrial Beatriz de la Fuente Casado. El valor medio de acuerdo (Kappa) fue de 0,875 , un dato que señala una adecuada fiabilidad para el análisis de las informaciones recogidas (Igartua, 2006; Neuendorf, 2002).

\section{Resultados}

Los seis periódicos analizados publicaron un total de 269 artículos informando sobre las elecciones autonómicas del 27 de septiembre de 2015, en los quince días que duró la campaña electoral. Esto es, cada periódico publicó, de media, tres noticias diarias en su portada sobre las elecciones, si bien se observaron grandes diferencias individuales. Así, El Punt Avui fue el medio que publicó mayor número de informaciones en primera página, un total de 76, seguido por El Periódico (66), El País (42), El Mundo (39) y La Vanguardia (36). A gran distancia el diario $A B C$ publicó "sólo" 10 informaciones en portada, pero hay que hacer constar el diferente diseño de este medio respecto al resto, pues como afirma su director, $A B C$ es "el único diario de toda la prensa española que comparece con portada y no con primera página" (Rubio, 2015), esto es, dedica gráfica y textualmente su primera página a un tema principal, en ocasiones único, o cuando menos muy dominante por encima de una o dos informaciones muy secundarias respecto a éste.

Sin embargo, este menor recuento cuantitativo, no supuso una menor importancia prestada a las elecciones autonómicas, ya que las diez ocasiones se trataron de 
la información principal de la portada. El Punt Avui y La Vanguardia dedicaron el principal espacio de su primera página en catorce ocasiones, El Periódico ocho, El País siete y El Mundo seis.

La importancia media de estas informaciones fue de $2,93(\mathrm{DT}=0,774)$ en una escala de 0 a 4, menor a la registrada en la consulta soberanista del 9-N, cuando alcanzó el 3,44 $(\mathrm{DT}=0,612)$, lo que reflejó diferencias significativas entre el índice de importancia de las informaciones de ambas convocatoria ( $U$ de Mann Whitney $=8274, p<0.001$ ). Sin embargo en las autonómicas de 2015, estos seis periódicos publicaron 269 noticias en portada, casi 18 por día, un número muy superior a las 6,9 noticias diarias que publicaron con ocasión del 9-N. El 27-S casi la mitad de las noticias (133), frente a sólo el 9\% en el 9-N, fueron artículos secundarios de los que tan sólo se publicaba el titular principal.

Así, no solo todos los diarios analizados informaron diariamente en portada, y habitualmente en el espacio principal, de las elecciones autonómicas, sino que además incluyeron otras piezas informativas de acompañamiento. Dos periódicos (El Periódico y El Punt Avui) avanzaron en su primera página el contenido de una serie de secciones diarias sobre la campaña electoral. Por ejemplo El Periódico llevó todos los días en portada la sección "Con la independencia...", que en cada número respondía a un interrogante diferente del tipo "¿Estaremos en Europa?” (11/09/2015), “¿Cobraremos las pensiones?” (14/09/2015) o “Dónde jugaría el Barça?” (20/09/2015). Por su parte, El Punt Avui creó la sección "Els arguments del sí" que también avanzó a diario en su primera página, por ejemplo "Els catalans continuarem dins la UE" (15/09/2015), "Per garantir l'estat del benestar" (17/09/2015) o "L'estat, més atractiu per als inversors" (19/09/2015). El resto de diarios también acostumbraron a publicar en portada el titular, y a veces la firma, de otros artículos, como "El candidato de usar y tirar" de Ignacio Vidal-Folch (El Mundo, 22/09/2015), "Reconstruir el Estado" de Juan Luis Cebrián (El País, 20/09/2015) o "La ANC se prepara para actos de desobediencia" (La Vanguardia, 13/09/2015).

Por tanto, el índice de importancia podía interpretarse de un modo equivocado, por lo que para comparar ambas convocatorias electorales se eliminaron este tipo informaciones secundarias, ya que no restaban importancia real a la cobertura del diario, sino que incluso la aumentaban. Resultaron entonces índices de importancia semejantes en ambos casos, 3,44 (DT =0,612) en el 9-N y 3,40 $(\mathrm{DT}=0,702)$ en el 27-S, sin que la prueba U de Mann Whitney reflejase diferencias significativas.

De este modo, lo habitual en la consulta del 9-N fue que los medios le dedicaran un único artículo al día en primera página, que solía ser de máxima o alta importancia, mientras que en el 27-S estos periódicos pasaron a informar en portada sobre las elecciones autonómicas en casi tres artículos diarios, dos de ellos de máxima o alta importancia y uno más de importancia media.

El 81,5\% de las noticias se refirieron temáticamente a la independencia, frente al $18,5 \%$ que no lo hicieron, informando de temas como la corrupción, el paro, la educación o las encuestas electorales. Si bien la prueba T de Student sólo mostró diferencias tendenciales en el índice de importancia entre unas y otras $(\mathrm{p}=0,053)$, los residuos corregidos mostraron una significativa mayor frecuencia de la esperada de las noticias de importancia máxima cuando se referían a la independencia (Prueba exacta de Fisher $=10,019, \mathrm{p}<0.01$ ).

El hecho noticioso también estuvo asociado estadísticamente con la referencia a la independencia (Prueba exacta de Fisher $=48,402, p<0.001$ ). Las casillas que mostraron mayores diferencias entre los valores esperados y los observados corres- 
pondieron a las acciones políticas de las instituciones españolas y de los políticos internacionales, que se relacionaron en todos los casos con la independencia.

Si bien no se halló asociación entre la referencia a la independencia y la Comunidad Autónoma en que se publicaba el medio, sí que fue significativa respecto a los periódicos concretos (Prueba exacta de Fisher $=32,915, \mathrm{p}<0.001$ ). Así, El Mundo (66,7\%), La Vanguardia $(69,4 \%)$ y El Periódico $(71,2 \%)$ informaron de la campaña electoral refiriéndose a la independencia en valores menores de los esperados, mientras que sólo El Punt Avui $(97,4 \%)$ se refirió a ésta en un mayor número de ocasiones a las esperadas. $A B C(90 \%)$ y El País $(92,1 \%)$ se quedaron en valores intermedios.

Resaltó el uso como fuente de los políticos catalanes $(21,89 \%)$ y españoles $(9,06 \%)$ o una mezcla de ambos $(12,08 \%)$, seguidos por las fuentes económicas $(11,32 \%)$, así como de políticos o personalidades internacionales (10,94\%). La prueba del chi cuadrado mostró un uso diferente de las fuentes entre ambas convocatorias electorales $[\chi 2(11, \mathrm{~N}=361)=182,12, \mathrm{p}<0.01]$. Los residuos tipificados corregidos mostraron que el mayor cambio fue el menor uso como fuentes de las instituciones, que en el caso de las catalanas cayeron desde casi la mitad (48,96\%) en el 9-N a un menguado 2,26\% en el 27-S, mientras que las españolas lo hicieron desde el 16,67\% al 1,89\%. En cambio aumentó el empleo como fuentes de los políticos, y aparecieron dos nuevas fuentes apenas empleadas en 2014: las instancias económicas subieron del 2,08 al 11,32\% y los políticos internacionales del 0 al 10,94\%.

Los posibles problemas monetarios y de financiación de la independencia fueron debatidos por todos los medios, en titulares como "Los bancos consideran irse de Cataluña si hay independencia " (El País, 19/09/2015), "Solo Grecia paga más que Cataluña por su deuda" (El Mundo, 16/09/2015), "El Banco de España avisa de que la secesión puede llevar al "corralito"' $(A B C, 22 / 09 / 2015)$, "La banca toma partido contra la independencia" (La Vanguardia, 19/09/2015), "La alerta de corralito enciende la campaña" (El Periódico, 22/09/2015) o "El Banco de España ahora niega el corralito" (El Punt Avui, 24/09/2015).

El novedoso empleo de fuentes internacionales se debió, en su mayor parte, a las referencias al presidente norteamericano y a diferentes actores europeos. El 16 de septiembre la recepción de Obama al rey Felipe VI sirvió para que todos los periódicos, salvo El Punt Avui, coincidieran en titulares muy similares que destacaban la defensa por parte del estadounidense de "una España fuerte y unida". El Punt Avui optó por un titular más ambiguo, "Cataluña entra en la Casa Blanca", ampliado en un sumario donde se aclaraba que "Felipe VI lleva el caso catalán a Obama y tan sólo arranca un apoyo de compromiso hacia una 'España fuerte y unida"'.

Los principales sujetos de la información fueron las instituciones catalanas $(35,63 \%)$ y españolas $(10,73 \%)$, junto con los políticos catalanes $(19,92 \%)$ y españoles $(4,98 \%)$. Se comprobó la existencia de diferencias significativas entre los sujetos que protagonizaron las noticias de la consulta soberanista y de las elecciones autonómicas. Así, los residuos tipificados corregidos resaltaron el menor protagonismo ofrecido a los políticos catalanes el 27-S, que descendió en más de trece puntos, en contraste con el ascenso de los ciudadanos, que más que duplicaron su protagonismo, y la aparición de sujetos internacionales y económicos.

A pesar de que en ambas ocasiones las acciones políticas fueron preponderantes en porcentajes casi idénticos (78,93 frente a $79,19 \%)$, la prueba de chi cuadrado mostró diferencias estadísticas en las acciones representadas por la prensa en cada convocatoria $\left[\chi^{2}(4, N=361)=43,76, p<0.001\right]$, ya que en el $20 \%$ de ocasiones res- 
tantes sí se produjeron grandes cambios, significativos según los valores de los residuos. Así, las acciones económicas pasaron de un reducido 2,08\% en el 9-N a un $13,79 \%$ en el $27-\mathrm{S}$, mientras que las acciones ilegales se redujeron a una tercera parte, del 18,75 al 6,13\%. El resto de acciones, reivindicación social y vida cotidiana, sumaron en conjunto poco más del $1 \%$.

Algo similar ocurrió con la representación por parte de los periódicos de los principales hechos noticiosos: si bien los principales sucesos informativos (las acciones políticas de instituciones y políticos catalanes) apenas cambiaron o no lo hicieron de modo significativo, los cambios en otras noticias menos habituales produjeron diferencias significativas entre una y otra convocatoria electoral $[\chi 2(22, N=357)=58,43$, $\mathrm{p}<0.001]$. El principal cambio fue la casi desaparición de noticias en que se informaba de políticos catalanes realizando o proponiendo realizar delitos o acciones ilegítimas, que pasaron del $12,5 \%$ en el $9-\mathrm{N}$ a un $0,77 \%$ en el $27-\mathrm{S}$. La otra novedad importante fue el creciente protagonismo de las acciones económicas por parte de las instituciones catalanes que aumentó del 2 al 11\%. Constatar la aparición como hecho noticioso relevente de las acciones políticas de sujetos internacionales (Ver datos en tabla 1).

Tabla 1. Comparación de los principales hechos noticiosos. Elaboración propia. Nota: $N=357$. Los valores de las celdas representan porcentajes. Las diferencias son significativas a nivel $p<0,001$

\begin{tabular}{|c|c|c|c|c|c|}
\hline \multicolumn{3}{|c|}{ Hecho noticioso } & \multirow{2}{*}{$\begin{array}{c}\text { Elecciones } \\
\text { autonómicas } \\
27-\mathrm{S}\end{array}$} & \multirow{2}{*}{$\begin{array}{c}\text { Consulta } \\
\text { soberanista } \\
9-\mathrm{N}\end{array}$} & \multirow{2}{*}{ Total } \\
\hline Sujeto & Acción & & & & \\
\hline \multirow{2}{*}{$\begin{array}{l}\text { Institución } \\
\text { catalana }\end{array}$} & \multirow[b]{2}{*}{ Política } & $\%$ & 24,14 & 25,00 & \multirow[b]{2}{*}{24,37} \\
\hline & & $\begin{array}{c}\text { Residuos } \\
\text { corregidos }\end{array}$ & $-0,17$ & 0,17 & \\
\hline \multirow[b]{2}{*}{ Político catalán } & \multirow[b]{2}{*}{ Política } & $\%$ & 16,86 & 20,83 & \multirow[b]{2}{*}{17,93} \\
\hline & & $\begin{array}{l}\text { Residuos } \\
\text { corregidos }\end{array}$ & $-0,87$ & 0,87 & \\
\hline \multirow{2}{*}{$\begin{array}{l}\text { Institución } \\
\text { española }\end{array}$} & \multirow[b]{2}{*}{ Política } & $\%$ & 9,58 & 16,67 & \multirow[b]{2}{*}{11,48} \\
\hline & & $\begin{array}{l}\text { Residuos } \\
\text { corregidos }\end{array}$ & $-1,86$ & 1,86 & \\
\hline \multirow[b]{2}{*}{ Ciudadanos } & \multirow[b]{2}{*}{ Política } & $\%$ & 10,34 & 6,25 & \multirow[b]{2}{*}{9,24} \\
\hline & & $\begin{array}{l}\text { Residuos } \\
\text { corregidos }\end{array}$ & 1,18 & $-1,18$ & \\
\hline \multirow{2}{*}{$\begin{array}{l}\text { Institución } \\
\text { catalana }\end{array}$} & \multirow[b]{2}{*}{ Economía } & $\%$ & 11,11 & 2,08 & \multirow[b]{2}{*}{8,68} \\
\hline & & $\begin{array}{c}\text { Residuos } \\
\text { corregidos }\end{array}$ & 2,69 & $-2,69$ & \\
\hline \multirow{2}{*}{$\begin{array}{l}\text { Político } \\
\text { español }\end{array}$} & \multirow[b]{2}{*}{ Política } & $\%$ & 4,98 & 8,33 & \multirow[b]{2}{*}{5,88} \\
\hline & & $\begin{array}{l}\text { Residuos } \\
\text { corregidos }\end{array}$ & $-1,19$ & 1,19 & \\
\hline \multirow{2}{*}{$\begin{array}{l}\text { Institución } \\
\text { catalana }\end{array}$} & \multirow{2}{*}{ Delito } & $\%$ & 2,68 & 6,25 & \multirow[b]{2}{*}{3,64} \\
\hline & & $\begin{array}{c}\text { Residuos } \\
\text { corregidos }\end{array}$ & $-1,60$ & 1,60 & \\
\hline \multirow{2}{*}{$\begin{array}{c}\text { Político } \\
\text { internacional }\end{array}$} & \multirow[b]{2}{*}{ Política } & $\%$ & 3,83 & 0,00 & \multirow[b]{2}{*}{2,80} \\
\hline & & $\begin{array}{c}\text { Residuos } \\
\text { corregidos }\end{array}$ & 1,95 & $-1,95$ & \\
\hline \multirow[b]{2}{*}{ Político catalán } & \multirow[b]{2}{*}{ Delito } & $\%$ & 0,77 & 12,50 & \multirow[b]{2}{*}{3,92} \\
\hline & & $\begin{array}{c}\text { Residuos } \\
\text { corregidos }\end{array}$ & $-5,06$ & 5,06 & \\
\hline & & Total & 100 & 100 & 100 \\
\hline
\end{tabular}


La mayor parte de las informaciones sobre el 27-S, el 41,4\%, recibió una evaluación del acontecimiento principal neutra, seguida por un 33,7\% de noticias negativas y un $24,2 \%$ positivas. Esta distribución fue similar a la obtenida el $9-\mathrm{N}$, como confirmó la U de Mann Whitney con un valor de 12312, p=0.790.

A continuación se comprobó la existencia de asociación estadística entre la evaluación y el hecho noticioso (Prueba exacta de Fisher $=68,113, \mathrm{p}<0.001$ ), como ya ocurriera con las informaciones de la consulta de 2014. Las evaluaciones neutras fueron mayoría en casi todos los hechos noticiosos, y se halló un cierto equilibrio entre evaluaciones positivas y negativas para un grupo de hechos: las acciones políticas de instituciones catalanas, españolas e internacionales, y las acciones económicas de las instituciones catalanas (Ver datos en tabla 2). Un mayor desequilibrio se detectó en los casos de las acciones políticas de los políticos catalanes, mayoritariamente negativas (36,4\% frente a $11,4 \%)$, de los ciudadanos, mayoritariamente positivas $(48,1 \%$ frente a $3,7 \%$ ) y de los políticos españoles, habitualmente negativas $(23,1 \%$ frente a $7,7 \%)$.

Tabla 2. Evaluación de los principales hechos noticiosos relacionados con el 27-S. Elaboración propia. Nota: $N=261$. Los valores de las celdas representan porcentajes.

La asociación es significativa a nivel $p<0,001$.

\begin{tabular}{|l|l|c|c|c|c|}
\hline \multicolumn{2}{|c|}{ Hecho noticioso } & \multicolumn{3}{c|}{$\begin{array}{c}\text { Evaluación del } \\
\text { acontecimiento principal }\end{array}$} & \multirow{2}{*}{ Total } \\
\cline { 1 - 5 } Sujeto & $\begin{array}{c}\text { Principal acción } \\
\text { descrita }\end{array}$ & Negativa & $\begin{array}{c}\text { Neutra o } \\
\text { ambigua }\end{array}$ & Positiva & \\
\hline Institución catalana & Política & 31,7 & 36,5 & 31,7 & 100 \\
\hline Político catalán & Política & 36,4 & 52,3 & 11,4 & 100 \\
\hline Institución catalana & Economía & 31 & 41,4 & 27,6 & 100 \\
\hline Ciudadanos & Política & 3,7 & 48,1 & 48,1 & 100 \\
\hline Institución española & Política & 36 & 24 & 40 & 100 \\
\hline Político español & Política & 23,1 & 69,2 & 7,7 & 100 \\
\hline Político internacional & Política & 20 & 50 & 30 & 100 \\
\hline Institución catalana & Delito & 100 & 0 & 0 & 100 \\
\hline
\end{tabular}

Se pudo comprobar una diferente evaluación del hecho principal en función de la Comunidad Autónoma en que se publicará El Periódico (U de Mann-Whitney = 4808, $\mathrm{p}<0.001$ ), de modo similar a lo descrito para la consulta soberanista de 2014. Los medios madrileños evaluaron las noticias de las elecciones autonómicas de un modo más negativo $(\mathrm{M}=1,58, \mathrm{DT}=0,683)$, que los medios catalanes, cuya media se mantuvo en torno al valor propio a una evaluación neutra $(\mathrm{M}=2,07, \mathrm{DT}=0,749)$. Para poder profundizar en estos datos se elaboraron tablas de contingencia que mostraran la evaluación de cada uno de los principales hechos noticiosos por parte de los periódicos de cada Comunidad Autónoma. De este modo se pudo comprobar que los medios de Madrid evaluaron de un modo mayoritariamente negativo tanto las acciones políticas de los políticos catalanes (71,4\% de las ocasiones) como de las instituciones catalanas $(66,7 \%)$. En cambio, las acciones políticas de las instituciones españolas fueron evaluadas de modo positivo en un $45,5 \%$ de veces, y las de los políticos españoles lo fueron de modo neutral $(83,3 \%)$ o positivo $(16,7 \%)$, nunca de 
modo negativo. La asociación resultó estadísticamente significativa (Prueba exacta de Fisher $=53,862, \mathrm{p}<0,001)($ Ver datos en tabla 3$)$.

Tabla 3. Evaluación de los principales hechos noticiosos relacionados con el 27-S por parte de los medios madrileños. Elaboración propia. Nota: $N=83$. Los valores de las celdas representan porcentajes. La asociación es significativa a nivel $* * * p<0,001$.

\begin{tabular}{|l|l|r|r|r|c|}
\hline \multicolumn{2}{|c|}{ Hecho noticioso } & \multicolumn{3}{c|}{$\begin{array}{c}\text { Evaluación del } \\
\text { acontecimiento principal }\end{array}$} & \multirow{2}{*}{ Total } \\
\hline \multicolumn{1}{|c|}{ Sujeto } & $\begin{array}{c}\text { Principal acción } \\
\text { descrita }\end{array}$ & Negativa & $\begin{array}{c}\text { Neutra o } \\
\text { ambigua }\end{array}$ & Positiva & \\
\hline Político catalán & Política & 71,4 & 28,6 & 0 & 100 \\
\hline Institución catalana & Política & 66,7 & 25 & 8,3 & 100 \\
\hline Institución española & Política & 18,2 & 36,4 & 45,5 & 100 \\
\hline Institución catalana & Delito & 100 & 0 & 0 & 100 \\
\hline Institución catalana & Economía & 100 & 0 & 0 & 100 \\
\hline Político español & Política & 0 & 83,3 & 16,7 & 100 \\
\hline Ciudadanos & Política & 0 & 80 & 20 & 100 \\
\hline \multicolumn{2}{|c|}{ Total } & 53,0 & 36,1 & 10,8 & 100 \\
\hline
\end{tabular}

La evaluación de los principales hechos noticiosos por parte de los medios catalanes también resultó estadísticamente significativa (Prueba exacta de Fisher $=50,065$, $\mathrm{p}<0.01$ ), y muy diferente a la descrita para los medios madrileños. Así, la evaluación neutra fue mayoritaria en la representación tanto de las acciones de políticas de instituciones $(39,3 \%)$ y políticos catalanes $(63,3 \%)$, como de las actuaciones económicas de las instituciones catalanas (52,2\%). Por el contrario, las acciones políticas de instituciones y políticos españoles fueron evaluadas más frecuentemente de modo negativo que positivo. Contra esta tendencia a evaluar más favorablemente los hechos protagonizados por sujetos del mismo área geográfica que el medio que los publica, resalta que las acciones políticas de los políticos catalanes fue representada por los periódicos catalanes más habitualmente de modo negativo (20\%) que positivo (16,7\%) (Ver datos en tabla 4).

Tabla 4. Evaluación de los principales hechos noticiosos relacionados con el 27-S por parte de los medios catalanes. Elaboración propia. Nota: $N=178$. Los valores de las celdas representan porcentajes. La asociación es significativa a nivel $* p<0,01$.

\begin{tabular}{|c|c|c|c|c|c|}
\hline \multicolumn{2}{|c|}{ Hecho noticioso } & \multicolumn{3}{|c|}{$\begin{array}{c}\text { Evaluación del } \\
\text { acontecimiento principal }\end{array}$} & \multirow{2}{*}{ Total } \\
\hline Sujeto & $\begin{array}{c}\text { Principal acción } \\
\text { descrita }\end{array}$ & Negativa & $\begin{array}{l}\text { Neutra } 0 \\
\text { ambigua }\end{array}$ & Positiva & \\
\hline Institución catalana & Política & 23,5 & 39,2 & 37,3 & 100 \\
\hline Político catalán & Política & 20 & 63,3 & 16,7 & 100 \\
\hline Institución catalana & Economía & 13 & 52,2 & 34,8 & 100 \\
\hline Ciudadanos & Política & 4,5 & 40,9 & 54,5 & 100 \\
\hline Institución española & Política & 50 & 14,3 & 35,7 & 100 \\
\hline Político internacional & Política & 20 & 50 & 30 & 100 \\
\hline Político español & Política & 42,9 & 57,1 & 0 & 100 \\
\hline \multicolumn{2}{|c|}{ Total } & 24,7 & 43,8 & 31,5 & 100 \\
\hline
\end{tabular}




\section{Conclusiones}

Los periódicos trataron todavía con mayor importancia las elecciones autonómicas catalanas del 27 de septiembre de 2015 que la consulta soberanista del 9 de noviembre de 2014, al publicar en portada un mayor número de informaciones relacionadas con la cita electoral. Si la información sobre la consulta de 2014 apareció todos los días en un artículo de máxima importancia de todos los periódicos analizados, la cobertura informativa en portada de las elecciones autonómicas de 2015 se amplió a unos tres artículos por medio y día, dos de máxima o alta importancia y uno de importancia media. La independencia de Cataluña se trata por tanto de un tema que amenaza con saturar por completo el espacio informativo prioritario de los periódicos españoles, estando a un paso de convertirse en tema único de portada durante la campaña electoral de 2015.

En respuesta a la primera pregunta de investigación en las elecciones autonómicas de 2015 se emplearon dos nuevas fuentes relevantes: las económicas y las internacionales. Los líderes de la Unión Europea y los Estados Unidos fueron representados para mostrar su disconformidad con la independencia, mientras que las fuentes económicas advirtieron de las consecuencias negativas del proceso soberanista.

En cuanto a la segunda pregunta planteada los principales cambios fueron la menor representación de acciones políticas de las instituciones españolas y delictivas de los políticos catalanes, frente al aumento de las acciones económicas de las instituciones catalanas y políticas de los políticos internacionales. Este resultado era esperable, pues la consulta de 2014 fue desestimada legalmente desde un principio por el Tribunal Constitucional, Gobierno central y buena parte de los políticos y medios de comunicación españoles, mientras que las elecciones autonómicas forman parte de la rutina democrática, incluso su carácter anticipado.

La evaluación de estos hechos noticiosos fue similar en ambas convocatorias electorales, existiendo en ambas ocasiones una asociación entre una determinada evaluación y un suceso informativo. Por un lado se detectó una evaluación neutra y equilibrada en las acciones políticas y económicas de las instituciones, fueran éstas catalanas, españolas o internacionales. Por otro, se comprobó una mayor frecuencia de evaluaciones negativas de las acciones políticas de los políticos catalanes y españoles, y positivas de los ciudadanos.

Igualmente, el tratamiento informativo de la campaña del 27 de septiembre de 2015 volvió a mostrar, como ya lo hiciera el 9-N de 2014, que un mismo hecho informativo era evaluado de modo diferente en función de la procedencia del medio de comunicación. Los medios madrileños representaron la campaña con matices más negativos, frente a la evaluación más neutra de los medios catalanes. De nuevo se volvió a detectar que los medios tendían a evaluar más positivamente las acciones de los sujetos de su misma procedencia geográfica y más negativamente a los sujetos más lejanos. Así los medios madrileños representaron mayoritariamente de modo negativo a los sujetos catalanes y positivo a los españoles, mientras que los medios catalanes hicieron lo inverso.

Por tanto podemos afirmar que han coexistido una serie de coincidencias y de diferencias entre el enmarcado informativo de las elecciones autonómicas de 2015 y el de la consulta soberanista de 2014. Si bien se mantiene entre ambas convocatorias electorales la preponderancia de las fuentes y del protagonismo de los sujetos políticos e institucionales, en el caso de las elecciones autonómicas aparecieron nuevas 
fuentes y sujetos, especialmente los actores internacionales y económicos. Si en la consulta del 9-N gran parte del debate mediático giró en torno a su carácter legal o ilegal, en el caso de las elecciones autonómicas el centro de atención se enfocó sobre las consecuencias económicas y de encaje internacional de la independencia.

Por tanto, puede afirmarse que el carácter plebiscitario de las elecciones autonómicas fue plenamente asumido por los medios de comunicación, que centraron el debate en la cuestión independentista en más del $80 \%$ de las noticias analizadas, en vez de en las competencias propias del Gobierno autonómico que se eligiera. Por todo ello, los partidos independentistas (CDC, ERC y CUP) lograron imponer el tema de debate público que les interesaba, consiguiendo que la campaña electoral se disputase en el campo de juego por ellos elegido y dispuesto. La "batalla de enmarcado" en que los partidos procuran imponer su diagnóstico de la situación (Miller y Riechert, 2001) se habría decantando en favor del bando independentista, que habría conseguido, cual flautista de Hamelín, que la atención mediática y el debate político girase en torno a la partitura soberanista.

También volvió a repetirse el carácter nacionalista de los medios de comunicación que informaron en favor de las posturas políticas mayoritarias en su área de procedencia. Se mantienen dos representaciones mediáticas antagónicas de unos mismos hechos a un lado y otro de la frontera autonómica de Cataluña con el resto de España, como ya se pusiera de manifiesto en el 9-N (Ballesteros, 2015a y 2015b), volviendo a revelarse dos "patrones específicos" que agrupan las variables de un modo sistemático, siguiendo los términos de Matthes y Kohring (2008). "De modo simbólico, si las fronteras geográficas aún no separan naciones de derecho sí que parecen determinar naciones informativas de hecho, caracterizadas por aportar interpretaciones propias e incompatibles de los mismos procesos políticos" (Ballesteros, 2015b: 180). Nos encontramos así con la construcción de muy diferentes, e incompatibles, marcos informativos sobre la cuestión catalana, cuyas repercusiones sobre las opiniones y actitudes de las audiencias en tanto ciudadanos y sujetos políticos deberán ser estudiadas en posteriores investigaciones.

\section{Referencias bibliográficas}

AIMC (2015): "Resumen general de resultados EGM. Febrero a Noviembre de 2015": http:// www.aimc.es/spip.php?action=acceder_document\&arg=2974\&cle=08efe06e $64115 \mathrm{e} 74$ ee48bd42eafc913eef981c8c\&file $=$ pdf\% $\%$ Fresumegm315.pdf [Fecha de consulta: 12 de febrero de 2016]

Ballesteros, Carlos (2014): "El enmarcado informativo del cannabis: Un estudio cuantitativo exploratorio desde la teoría del framing". Anuario Electrónico De Estudios En Comunicación Social "Disertaciones", 7 (1), 67-103: http://revistas.urosario.edu.co/ index.php/disertaciones/article/view/3840.pdf. [Consulta: 2 de enero de 2016]

Ballesteros, Carlos; Dader, José Luis; y Muñiz, Carlos (2015): "Los marcos informativos del cannabis en la prensa española. evidencia empírica desde la teoría del framing". ZerRevista de Estudios de Comunicación, 20, n 38, 67-85: http://www.ehu.eus/ojs/index. php/Zer/article/view/14782/13062 [Consulta: 12 de enero de 2016]

Ballesteros, Carlos (2015a): "Naciones mediáticas. Los marcos informativos de la consulta sobre la independencia de cataluña (9-N)". Doxa Comunicación. Revista Interdisciplinar de Estudios de Comunicación y Ciencias Sociales, 20, 162-83. 
Ballesteros, Carlos (2015b): "El desafío inadvertido. la consulta sobre la independencia de Cataluña desde el marco informativo del conflicto". Anàlisi, 20, 48-64: doi: 10.7238/a. v0i53.2531 [Consulta: 2 de enero de 2016]

Bryant, Jennings y Miron, Dorina (2004): "Theory and research in mass Communications". Journal of Communication, 4, 54, 662-704: doi: 10.1111/j.1460-2466.2004.tb02650.x [Consulta: 18 de enero de 2016]

Cacciatore, Michael; Scheufele, Dietram; and Iyengar, Shanto (2016): "The end of framing as we know it ... and the future of media effects". Mass Communication and Society, 19 (1), 7-23: doi: 10.1080/15205436.2015.1068811 [Consulta: 4 de diciembre de 2015]

Catalunya Sí Que Es Pot (2015): "El programa de la gent. Catalunya sí que es pot”: http:// catalunyasiqueespot.cat/wp-content/uploads/2015/09/CATSIQUESPOT_programa_10Definitiu-2015-9-11.pdf [Consulta: 1 de diciembre de 2015].

CUP (2015): "Programa polític per a les eleccions al Parlament de Catalunya del 27 de setembre de 2015":

http://cup.cat/sites/default/files/programa_de_la_cup_crida_constituent_27-S.pdf [Consulta: 1 de diciembre de 2015]

D’Angelo, Paul (2002): "News framing as a multi-paradigmatic research program: A response to Entman". Journal of Communication, 52, 870-88: doi: 10.1111/j.1460-2466.2002. tb02578.x [Consulta: 22 de noviembre de 2015]

Decreto 174/2015, de 3 de agosto, de convocatoria de elecciones al Parlamento de Cataluña y de su disolución.

https://www.boe.es/boe/dias/2015/08/04/pdfs/BOE-A-2015-8776.pdf [Consulta: 4 de diciembre de 2015]

Entman, Robert (1993): "Framing: Toward clarification of a fractured paradigm. Journal of Communication, 43 (4), 51-8.

Pérez, David (2014): “¿Qué son las elecciones plebiscitarias?”, en EITB, 14 de octubre: http:// www.eitb.eus/es/noticias/politica/detalle/1428428/elecciones-plebiscitarias-catalunaque-son-proceso-consecuencias/ [Consulta: 4 de noviembre de 2015]

El Confidencial (2015): "División en la política catalana sobre el carácter plebiscitario del 27-S", 3 de agosto:

http://www.elconfidencial.com/espana/cataluna/2015-08-03/division-en-la-politicaobre-el-caracter-plebiscitario-del-27-s_954421/ [Consulta: 8 de diciembre de 2015]

EFE(2015): "Mas convoca mañana elecciones entre la división sobre su carácter plebiscitario", 2 de agosto: http://www.efe.com/efe/espana/politica/mas-convoca-manana-eleccionesentre-la-division-sobre-su-caracter-plebiscitario/10002-2679334\# [Consulta: 8 de diciembre de 2015]

García, Antia (2015): "La oposición niega el carácter plebiscitario de las elecciones del 27S", en EL PAÍS, 4 de agosto:

http://ccaa.elpais.com/ccaa/2015/08/04/catalunya/1438710216_430455.html [Consulta: 9 de diciembre de 2015]

Gamson, William y Modigliani, Andre (1987): "The changing culture of affirmative action". Research in Political Sociology, 3, 137-77.

Gitlin, Todd (1980): The whole world is watching: Mass media in the making and unmaking of the new left. Berkeley. Berkeley, University of California Press.

Goffman, Erving (1974): Frame analysis. an essay on the organization of experience. New York, Harper Colphon.

Igartua, Juan José (2006): Métodos cuantitativos de investigación en comunicación. Barcelona, Bosch. 
Igartua, Juan José; Muñiz, Carlos; y Cheng, Lifen (2005): “La inmigración en la prensa española. Aportaciones empíricas y metodológicas desde la teoría del encuadre noticioso". Migraciones, 0, 17, 143-81:

http://revistas.upcomillas.es/index.php/revistamigraciones/article/view/4220/4043 [Consulta: 16 de diciembre de 2015]

Iyengar, Shanto (1991): Is anyone responsible? How television frames political issues. Chicago, University of California Press.

Junts Pel Sí (2015): "Programa electoral", en:

http://juntspelsi.s3.amazonaws.com/assets/150905_Programa_electoral_v1.pdf [Consulta: 1 de diciembre de 2015]

Lind, Rebbeca \& Salo, Colleen (2002): "The framing of feminists and feminism in news and public affairs programs in US electronic media". Journal of Communication, 52 (1), 211-28.

Luhmann, Niklas (2000): La realidad de los medios de masas. Barcelona, Anthropos.

Matthes, Jörg (2009): "What's in a frame? A content analysis of media framing studies in the world's leading communication journals, 1990-2005". Journalism \& Mass Communication Quarterly, 86 (2), 349-67: doi: 10.1177/107769900908600206 [Consulta: 1 de julio de 2015]

Matthes, Jörg \& Kohring, Matthias (2008): “The content analysis of media frames: Toward improving reliability and validity". Journal of Communication, 58 (2), 258-79: doi: doi:10.1111/j.1460-2466.2008.00384.x [Consulta: 1 de julio de 2015].

Miller, Mark \& Riechert, Bonnie (2001): "The spiral of opportunity and frame resonance. mapping the issue cycle in news and public dicourse", in REESE, Stephen; GANDY, Oscar; and GRANT, August: Framing public life: Perspectives on media and our understanding of the social world. New Jersey: Routledge, pp. 107-121.

Muñiz, Carlos (2015): "La política como debate temático o estratégico. Framing de la campaña electoral mexicana de 2012 en la prensa digital”. Comunicación y Sociedad, 23, 67-95: http://www.scielo.org.mx/pdf/comso/n23/n23a4.pdf [Consulta: 16 de noviembre de 2015]

Neuendorf, Kimberly (2002): Content analysis guidebook. Thousand Oaks, Sage Publications.

Neuman, Russell; Just, Marion; and CRIGLER, Ann (1992): Common knowledge. Chicago, University of Chicago Press.

Noguer, Miquel (2015): “Artur Mas: "El sí a la independencia ganará a partir de 68 diputados”, en EL PAÍS, 4 de agosto:

http://ccaa.elpais.com/ccaa/2015/08/04/catalunya/1438683546_309224.html [Consulta: 9 de diciembre de 2015]

Pan, Zhongdang \& Kosicki, Gerald (1993): "Framing analysis: An approach to news discourse". Political Communication, 10, 55-76.

Porto, Mauro (2002): "Framing the world of politics: How governmental sources shape the production and the reception of TF news in brazil". 23rd International Conference of the International Association for Media and Communication Research: http://www.unb.br/ fac/mporto/index.html [Consulta: 22 de noviembre de 2007]

PPC (2015): "Unidos ganamos. Plantemos cara. 100 propuestas de gobierno 2015": http://www.ppcatalunya.com/wp-content/uploads/programa_elecotrol_cast_2015.pdf [Consulta: 10 de noviembre de 2015]

PSC (2015): "Nuestro compromiso. Soluciones justas y acordadas. Programa electoral del PSC para las elecciones catalanas del 27 de septiembre de 2015": http://www.socialistes. 
cat/files/fitxers/download/4d3eabd33d38f173dddc4ca1fff73054 [Consulta: 22 de diciembre de 2015]

Riffe, Dan (2004): “An editorial comment”. Journalism \& Mass Communication Quarterly, $81(2), 232-233$.

Rubido, Bieito (2015): "Una fotografía de portada", en $A B C, 3$ de septiembre: http://www. $A B C$.es/medios/20150903/ABCi-foto-nino-siria-201509031229.html [Consulta: 8 de diciembre de 2015]

Sádaba, Teresa y Rodríguez Virgili, Jordi (2007): "La construcción de la agenda de los medios. El debate del Estatut en la prensa española". Ámbitos, 16, 187-211: http://www. redalyc.org/articulo.oa?id=16801610 [Consulta: 8 de enero de 2016]

Sastre, Daniel (2015) "Mas convoca a la "nación" el 27-S como "último recurso", en El Mundo, 3 de agosto, en:

http://www.elmundo.es/cataluna/2015/08/03/55bfa6f722601d79268b45a1.html [Consulta: 4 de diciembre de 2015]

Scheufele, Dietram \& Iyengar, Shanto (2014): The state of framing research: A call for new directions. Oxford Handbooks Online:

http:/www.oxfordhandbooks.com/view/10.1093/oxfordhb/9780199793471.001.0001/ oxfordhb-9780199793471-e-47 [Consulta: 15 de enero de 2016]

Semetko, Holli \& Valkenburg, Patty (2000): "Framing european politics: A content analysis of press and television news". Journal of Communication, 50 (2), 93-109.

Tàbara, Joan; Costejà, Meritxell; y Woerden, Fincent van (2004): "Las culturas del agua en la prensa española. los marcos culturales en la comunicación sobre el plan hidrológico nacional". Papers, 73, 153-79.

UNIÓ (2015). "Programa electoral. La Força del seny":

http://www.unio.cat/sites/all/themes/unio/images/PROGRAMA_ELECTORAL_ UDC_2015.pdf [Consulta: 20 de enero de 2016]

Valera, Lidia y García López, Guillermo (2014): “Agenda y marcos en las webs de PP y PSOE en la cibercampaña de 2011”. Revista Latina De Comunicación Social, 69, 41-66, en:

http://www.revistalatinacs.org/069/paper/1000_Valencia/03_Valera.html [Consulta: 10 de enero de 2016]

Weaver, David (2007): “Thoughts on agenda setting, framing, and priming”. Journal of Communication, 57 (1), 142-147.

Carlos A. Ballesteros Herencia Fernando es Doctor en Ciencias de la Información (2012), licenciado en Periodismo (1995) y grado en Ciencias del Deporte y la Actividad Física (2011). Es profesor en el Grado de Periodismo de la Universidad de Valladolid. Miembro del proyecto I+D+I "Las estrategias de campaña online de los partidos políticos españoles 2015-2016" (Facultad de Ciencias de la Información, UCM) y de los proyectos de Innovación Docente "Enseñanza en Igualdad e Inclusión de Género" y "Evaluación y desarrollo de metodologías docentes en comunicación y competencia mediática" (Universidad de Valladolid). Sus principales líneas de investigación son la representación de los marcos informativos (news frames) en los medios de comunicación, la responsabilidad de la prensa ante las drogas y el periodismo político. Ha sido director de La Voz de Albacete y El Espectador Crítico, responsable de Comunicación de UPA-Castilla-La Mancha y profesor de Educación Física. 